\title{
An Analysis of the Multivariate-repeated- measures-experiment Evaluating the Influence of Gibberellic Acid on Cherry Fruit Quality
}

\author{
Damijana Kastelec ${ }^{1}$ and Valentina Usenik ${ }^{2}$
}

\begin{abstract}
A repeated-measures experiment design including two between-unit factors and one within-unit factor was performed in order to examine the influence of gibberellic acid on cherry fruit quality. One of the two between-unit factors had two levels represented by two cherry cultivars ('Van', 'Sunburst') and the other consisted of two treatments: spraying with gibberellic acid and spraying with pure water as a control. An experimental unit was a cherry branch on which 20 cherries were sampled randomly and marked. The three variables which describe ripeness of cherries were measured over 6 time points at three or four day intervals during maturation. After picking the cherries, measurements of fruit firmness were made on 3 different sides of each marked cherry. The univariate and multivariate repeated measures ANOVA was used for the analysis of two sets of data.
\end{abstract}

\section{Introduction}

Fruit cracking, which is thought to result from increased turgor caused by water uptake into the fruit, is one of the major problems in cherry cultivation. Rainfall during the harvest season causes this fruit to crack and consequently the yield decreases. To prevent cracking, growers often prematurely pick cherries of lower fruit quality. It has been shown that the treatment of cherries with gibberellic acid (GA3) can reduce fruit cracking, increase firmness, weight and the content of soluble solids, and delay fruit softening and the time of ripening (Dermisoy \& Bilgener 1998, Facteau et al. 1985, Choi et al. 2002, Kappel and MacDonald 2002). To examine the influence of GA3 on ripening, firmness and resistance to cracking of cherries, an experiment was carried out in 2002 in the Bilje Fruit

\footnotetext{
${ }^{1}$ Biotechnical Faculty, University of Ljubljana, Slovenia; damijana.kastelec@bf.uni-lj.si

${ }^{2}$ Biotechnical Faculty, University of Ljubljana, Slovenia; valentina.usenik@bf.uni-lj.si
} 
Growing Centre near Nova Gorica (Slovenia). The two sweet cherry cultivars 'Van' and 'Sunburst', which are very common in Slovenian orchards in the Primorska region, were treated in the experiment. Some cherries of each cultivar were sprayed with GA3 and the others only with water. The colour of the cherries was measured 6 times during maturation to detect differences in ripening. After picking, fruit firmness and the cracking index were measured.

The experiment was set up as a factorial repeated measures experiment with three fixed and one nested random factor. The objective of this paper is a statistical analysis of the experiment.

The common characteristic of factorial repeated measures experiments is the observation of the same experimental unit over several treatments or at different points of time. Such experimental designs are very often used in agriculture, where a typical experimental unit is an animal, a plant, a plot, a fruit, etc. When comparing the means of the treatments applied to each experimental unit, we examine a factor called as the within-units factor. The factor whose levels determine the groups of experimental units that we wish to compare is the between-units factor.

In general two different approaches to the hypothesis testing have been used in the analysis of such experiments. One is a univariate mixed-model nested design, where a nested random factor is included in the model to absorb the correlation between the experimental errors (also known as a split-plot design) (Fisher, 1971). The assumptions, concerning univariate normal distribution in the underlying population, sphericity and compound symmetry of error variance-covariance matrix must be valid if the univariate analysis is appropriate. A more general approach is a multivariate analysis. It assumes a multivariate normal underlying distribution in the population, and the experimental errors are considered to be correlated. In the multivariate approach two methods can be used: MANOVA with the profile analysis and a completely unstructured error variance-covariance matrix, and Linear Mixed Models (LMM), whose advantage is that several different covariance structures can be estimated and the most appropriate one chosen. The above mentioned approaches lead to the same results when the sphericity and compound symmetry of error variance-covariance matrix is met.

\section{Experimental design}

The experiment was carried out on 7-year old 'Van' and 'Sunburst' sweet cherry trees. Among similar sized and oriented branches on the trees six branches were randomly selected for each cultivar. Then 20 cherries were sampled randomly and marked on each branch. This means that a two-stage hierarchical sampling was carried out and the branches represented the nested random factor. Three of the six branches were sprayed with GA3 while the other three branches were sprayed with water and were protected from spraying with GA3. GA3 was sprayed during the 
transition from green to straw-yellow fruit colour, about 7 weeks after full blossom.

The cultivars and GA3 treatment are two between-unit fixed factors and time is the within-unit or repeated factor.

Three variables: brightness, intensity of green-red colour (Green/Red) and intensity of blue-yellow colour (Blue/Yellow), were measured with a chromameter for each of the twenty cherries over 6 time points at three- or four-day intervals during maturation (20. 5. 2002, 23. 5. 2002, 27. 5. 2002, 30. 5. 2002, 3. 6. 2002 and 6. 6. 2002). After all the cherries from chosen branches were picked, at fruit maturity, among all the picked intact cherries from each branch 20 intact fruits were chosen for measurements of firmness on 3 different sides of the cherry. These cherries were not the same as those that were marked for colour variables measurements. The marked cherries were put into pure water (the water temperature was $24^{\circ} \mathrm{C}$ ) and the number of cracked cherries was counted after 4 and 6 hours for measurements of cracking index.

\section{Data}

After the first exploratory data analysis of colour variables, we had to eliminate the data for some cherries from the further analysis because it was evident that some larger and very evident measurement errors existed in the data. Thus, the number of 20 cherries per branch was reduced to 16 to 19 cherries per branch. The scheme of the data is presented in Table 1.

Table 1: The scheme of the data.

\begin{tabular}{|c|c|c|c|c|c|c|c|c|}
\hline & & & & Dat & & & & \\
\hline Treatment & Cultivar & Branch & Cherry fruit & $D_{1}$ & $D_{2}$ & $D_{3}$ & $D_{4}$ & $D_{5}$ \\
\hline \multirow{6}{*}{ Control } & \multirow{3}{*}{ 'Van' } & $b_{1}$ & $c_{1}, \ldots, c_{n_{1}}$ & & & & & \\
\hline & & $b_{2}$ & $c_{n_{1}+1}, \ldots, c_{n_{2}}$ & & & & & \\
\hline & & $b_{3}$ & & & & & & \\
\hline & \multirow{3}{*}{ 'Sunburst' } & $b_{4}$ & & & & & & \\
\hline & & $b_{5}$ & & & & & & \\
\hline & & $b_{6}$ & & & & & & \\
\hline \multirow{6}{*}{ GA3 } & \multirow{3}{*}{ 'Van' } & $b_{7}$ & & & & & & \\
\hline & & $b_{8}$ & & & & & & \\
\hline & & $b_{9}$ & & & & & & \\
\hline & \multirow{3}{*}{ 'Sunburst' } & $b_{10}$ & & & & & & \\
\hline & & $b_{11}$ & & & & & & \\
\hline & & $b_{12}$ & $c_{n_{11}+1}, \ldots, c_{n_{12}}$ & & & & & \\
\hline
\end{tabular}



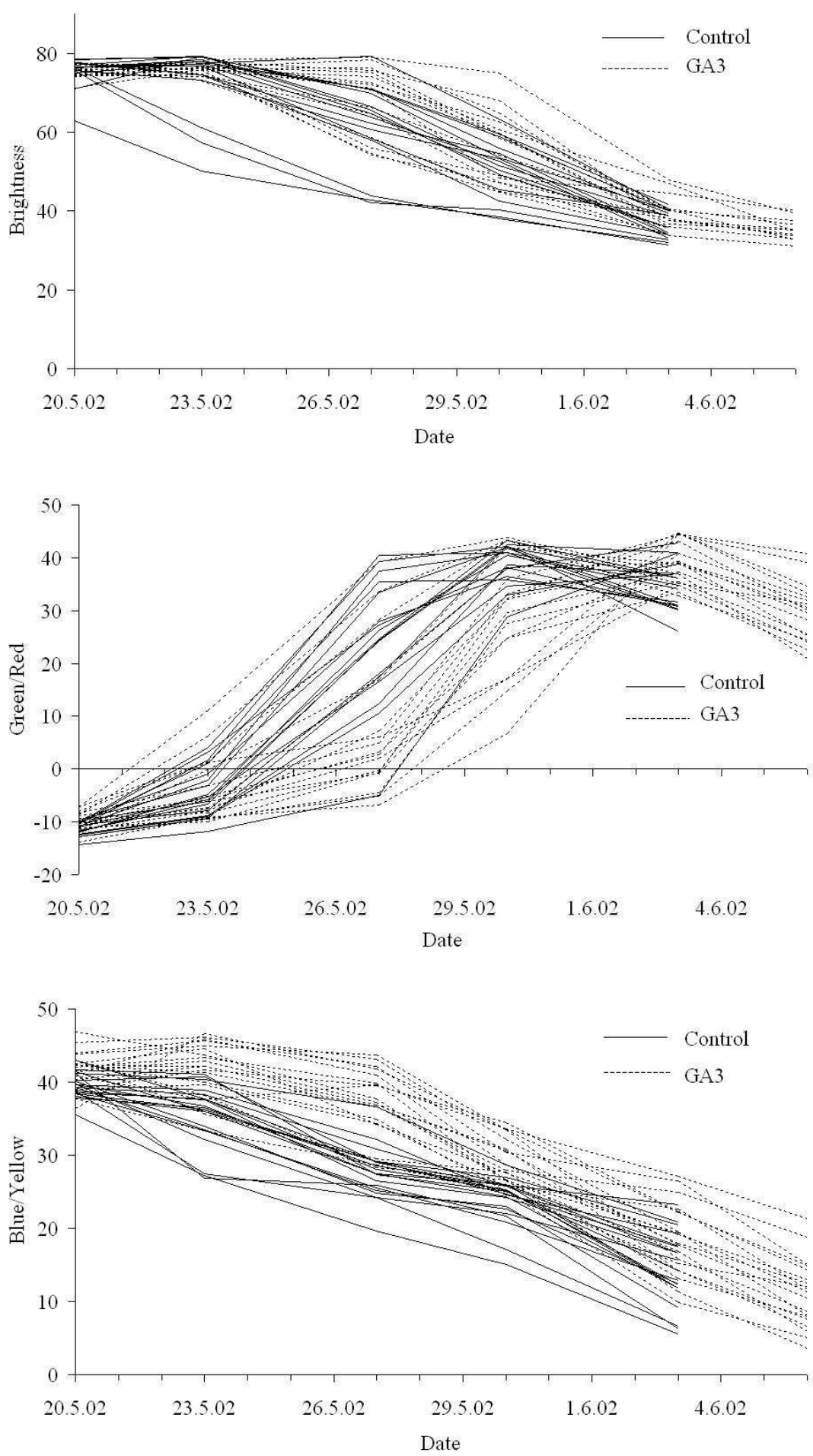

Figure 1: Time-related changes in brightness, green/red and blue/yellow for each cherry on one branch treated as Control and on one branch treated with GA3 in cv. 'Van'. 

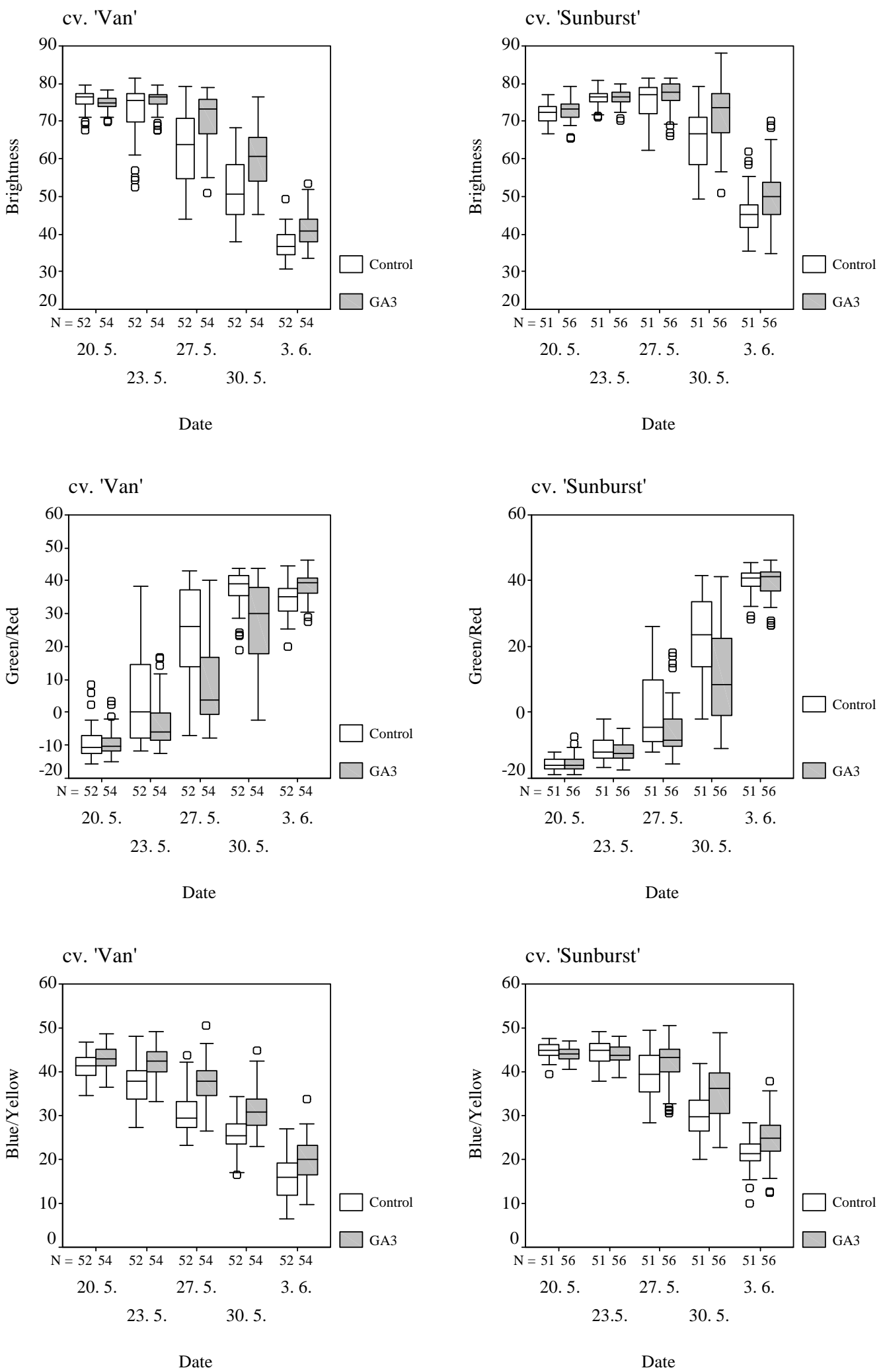

Figure 2: Box plots of brightness, green/red and blue/yellow for each treatment combination. 
Figure 1 presents the results of measuring the three colour variables of the cherries: brightness, green/red and blue/yellow, on all six consecutive dates for the cherries on just one branch for the cv. 'Van'. Any one line in the figure represents the values for one cherry.

The results of measuring the colour variables for all three branches together are presented with box-plots in Figure 2. Each box-plot presents the distribution of colour variable for the group of cherries on three branches for one treatment combination. In general, the brightness decreases during ripening, as do the blue/yellow values, while the green/red values increase. This means that cherries become darker, redder and yellower during maturation. For all three variables there is much variability between the cherry fruits and variability also changes with time. Quite notable differences exist between the two varieties: 'Van' starts to change colour earlier than 'Sunburst' does. On the basis of subjective decision of experts, the control cherries were mature before those treated with GA3 and were picked three days earlier in both varieties. Only five time points were taken into account in the further statistical analysis.

Figure 3 displays the distributions of fruit firmness for each treatment combination. In general, the values are greater for all three sides for the cherries treated with GA3 in both varieties, but in the case of 'Sunburst' there exists much larger variability of data for the cherries treated with GA3. This difference in variability should be taken into account in the further analysis.
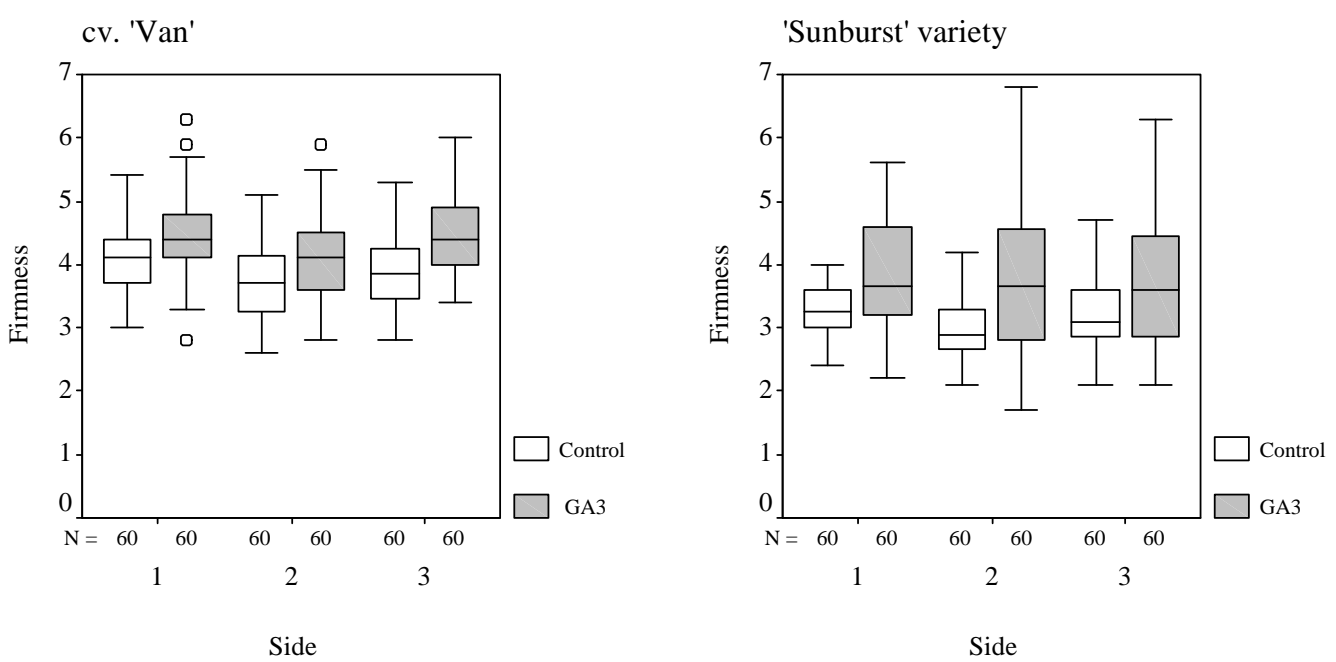

Figure 3: Box plots of fruit firmness for each treatment combination. 


\section{The mathematical models and statistical inference}

Using the statistical analysis of the two between-unit factors and one repeated factor experiment, we would like to answer the following questions:

- How does the colour of cherries change with time?

- What influence does GA3 have on the colour of cherry fruits?

- Is the time related change of cherry colour the same irrespective of whether they have or have not been sprayed with GA3 ?

- Are there differences in colour between the two varieties?

- Do the differences in colour between the cultivars change with time?

- Is the GA3 influence on colour the same in both cultivars?

- Does the GA3 influence on colour change with regard to both the cultivar and the time?

The null hypothesis which we want to test for each of the three variables concerning the colour of cherries (brightness, green/red, blue/yellow) measured at 5 time points are:

$H_{0}$ : There is no main effect of the $G A 3$ treatment.

$H_{0}: \mu_{G A 3}=\mu_{C}$.

$H_{0}$ : There is no main effect of the time.

$H_{0}: \mu_{D 1}=\mu_{D 2}=\mu_{D 3}=\mu_{D 4}=\mu_{D 5}$

$H_{0}$ : There is no main effect of the cultivar.

$H_{0}: \mu_{\text {'Van' }}=\mu_{\text {'Sunburst }}$

$H_{0}$ : There is no interaction between the time and the GA3 treatment.

$H_{0}$ : There is no interaction between the time and the cultivar.

$H_{0}$ : There is no interaction between the GA3 treatment and the cultivar.

$H_{0}$ : There is no interaction between the time, the GA3 treatment and the cultivar.

Similar questions and hypotheses could be stated for the firmness variable, measured with a penetrometer on three different sides of the cherries, but instead of the repeated factor time factor side of the measurement is now repeated.

Although there exist some outliers and asymmetry in empirical distributions of our data, we assumed that the three colour-variables and fruit firmness had a normal distribution in the underlying population of cherries. In the mathematical model we need to consider that the measurements on the same experimental unit at different time points are correlated. First, the univariate ANOVA mathematical model, in which all factors and their interactions can be explained in transparent way, will be presented and used for the fruit firmness analysis. Secondly, the MANOVA approach will be presented and used for the colour variables analysis. 


\subsection{The univariate approach}

In the univariate approach the cherry branches and fruits are regarded in the model as nested random factors (a kind of dummy variable) to absorb the correlation between experimental errors. This approach has the advantage of permitting a relatively simple derivation of the expected values of the mean squares, it also has grater power than the multivariate test.

In the mathematical model three fixed factors: GA3 treatment, cultivar and repeated factor time will be included together with two nested random factors: branch as an experimental first order unit and cherry as an experimental second order unit. The mathematical model for these three factor repeated measures is

$$
y_{i j k l m}=\mu+\tau_{i j}+b_{(i j) k}+c_{(i j k) l}+\gamma_{m}+(\tau \gamma)_{i j m}+(\not b)_{(i j) k m}+(\gamma c)_{(i j k) l m}
$$

where

$$
\tau_{i j}=\alpha_{i}+\beta_{j}+(\alpha \beta)_{i j} \quad \text { and } \quad(\tau \gamma)_{i j m}=(\alpha \gamma)_{i m}+(\beta \gamma)_{j m}+(\alpha \beta \gamma)_{i j m}
$$

$\alpha_{i}$ represents the main effect of fixed factor GA3 treatment, $i=1, \ldots, a, a=2 ; \beta_{j}$ represents the main effect of fixed factor cultivar, $j=1, \ldots, b, b=2 ; \alpha \beta_{i j}$ represents the interaction effect of fixed factors GA3 treatment and cultivar; $b_{(i j) k}$ represents the random effect of branch, $k=1, \ldots, b_{r}, b_{r}=3$ is number of branches nested in each treatment combination; $c_{(i j k) l}$ represents the random effect of cherries, $l=1, \ldots, n_{i j k}, \quad n_{i j k}$ (between 16 and 20) number of second order experimental units (cherries) within each level of random first order factor (branch); $\gamma_{m}$ represents the main effect of repeated factor time, $m=1, \ldots, d, d=5$; $(\alpha \gamma)_{\text {im }}$ represents the effect of interaction between GA3 treatment and time; $(\beta \gamma)_{j m}$ represents the effect of interaction between cultivar and time; $(\alpha \beta \gamma)_{i j m}$ represents the effect of interaction between GA3 treatment, cultivar and time; $(\not b)_{(i j) k m}$ and $(\gamma c)_{(i j k) l m}$ represents the interaction between time and the experimental units of first and second order, respectively.

The classical ANOVA assumptions are:

$$
\begin{aligned}
& \sum_{i} \alpha_{i}=\sum_{j} \beta_{j}=\sum_{m} \gamma_{m}=0, \\
& \sum_{i} \alpha \beta_{i j}=\sum_{j} \alpha \beta_{i j}=\sum_{i} \alpha \gamma_{i m}=\sum_{j} \beta \gamma_{j m}=\sum_{m} \alpha \gamma_{i m}=\sum_{m} \beta \gamma_{j m}=\mathbf{0}, \\
& \sum_{i} \alpha \beta \gamma_{i j m}=\sum_{j} \alpha \beta \gamma_{i j m}=\sum_{m} \alpha \beta \gamma_{i j m}=0, \\
& (\not b)_{(i j) k m} \sim N\left(0, \sigma_{b}^{2}\right) \text { and }(\gamma)_{(i j k) l m} \sim N\left(0, \sigma_{c}^{2}\right) .
\end{aligned}
$$


The univariate ANOVA table is presented in Table 2. For simplicity of the notation in the table, it is assumed that $n_{i j k}=n$ for all the branches. This is not true in our case, but incorporating a different $n_{i j k}$ in the calculation of appropriate mean sums of squares causes no difficulties.

Table 2: ANOVA table for the univariate approach for two between-unit and one repeated factor with two nested random factors in the case when there is equal number of cherries $n$ on all branches.

\begin{tabular}{|c|c|c|c|}
\hline Source of variation & Degrees of freedom & MS & $\mathrm{F}$ \\
\hline \multicolumn{4}{|l|}{ Between units } \\
\hline GA3 & $a-1=1$ & MSA & MSA/MSEB \\
\hline Cultivar & $(b-1)=1$ & MSB & MSB/MSEB \\
\hline GA3*Cultivar & $(a-1)(b-1)=1$ & MSAB & MSAB/MSEB \\
\hline Branches within $G A 3 *$ Cultivar & $a b\left(b_{r}-1\right)=8$ & MSEB & \\
\hline Cherries within (Branches and $G A 3 *$ Cultivar) & $a b b_{r}(n-1)=228$ & MSEBC & \\
\hline \multicolumn{4}{|l|}{ Within units } \\
\hline Time & $d-1=4$ & MSD & MSD/MSE \\
\hline Time *GA3 & $(d-1)(a-1)=4$ & MSDA & MSD/MSE \\
\hline Time ${ }^{*}$ Cultivar & $(d-1)(b-1)=4$ & MSDB & MSDB/MSE \\
\hline Time ${ }^{*} G A 3 *$ Cultivar & $(d-1)(a-1)(b-1)=4$ & MSDAB & MSDAB/MSE \\
\hline Time*(branches within $G A 3^{*}$ Cultivar) & $a b\left(b_{r}-1\right)(d-1)=32$ & MSE & \\
\hline $\begin{array}{l}\text { Time* }(\text { cherries within } \quad \text { (branches and } \\
\left.\text { GA } 3^{*} \text { Cultivar }\right)\end{array}$ & $a b b_{r}(n-1)(d-1)=908$ & MSEC & \\
\hline Total & $a b b_{r} d n-1=1199$ & & \\
\hline
\end{tabular}

The univariate ANOVA has been found appropriate if the error variancecovariance matrix for the repeated factor has a special structure called compound symmetry. It allows for the variables to be correlated but restricts every variable having the same variance and every pair of variables having the same covariance. In the case of compound symmetry, colour variables under any two dates should be equally correlated and have the same variance. On the basis of the exploratory data analysis it is hard to believe that this can be assumed. But we could assume compound symmetry for the firmness of fruits. The Mauchly's test was used to test the structure of the variance-covariance matrix and it showed that the assumption of compound symmetry held true for firmness but not for the three colour variables. 


\subsection{The multivariate approach}

In the multivariate approach, the $d$ responses $\mathbf{y}_{i j k l}=\left[y_{i j k l 1}, y_{i j k l 2}, \ldots, y_{i j k l d}\right]$ (repeated measures) for each experimental unit constitute a vector $\mathbf{y}_{i j k l}$. The mathematical model (6.1) is now written in matrix form as a simple two-way MANOVA model:

$$
\mathbf{y}_{i j k l}=\boldsymbol{\mu}+\boldsymbol{\alpha}_{i}+\boldsymbol{\beta}_{j}+\boldsymbol{\alpha} \boldsymbol{\beta}_{i j}+\boldsymbol{\varepsilon}_{i j k l}
$$

where $\boldsymbol{\alpha}_{i}$ is the effect of the $i$ th level of GA3 treatment on each of the $d$ variables in $\mathbf{y}_{i j k l}, \boldsymbol{\beta}_{j}$ is the effect of the $j$-th level of cultivar, and $\boldsymbol{\alpha}_{i j}$ is the interaction effect, $\boldsymbol{\varepsilon}_{i j k l}$ is error vector which is further partitioned into two parts corresponding to experimental units of the first and of the second order. We use conditions $\sum_{i} \boldsymbol{\alpha}_{i}=\sum_{j} \boldsymbol{\beta}_{j}=\sum_{i} \boldsymbol{\alpha} \boldsymbol{\beta}_{i j}=\sum_{j} \boldsymbol{\alpha} \boldsymbol{\beta}_{i j}=\mathbf{0}$ and assume that $\boldsymbol{\varepsilon}_{i j k l} \sim N_{d}(\mathbf{0}, \boldsymbol{\Sigma})$.

This model seems to involve only factors GA3 treatment and cultivar and their interaction but an approach similar to the profile analysis in the classical MANOVA can be used to obtain tests on factor Time and the interactions time*GA3, time* ${ }^{*}$ cultivar and time ${ }^{*} G A 3 *$ cultivar (Rencher, 1995). The MANOVA assumption that $\operatorname{cov}\left(\mathbf{y}_{i j k l}\right)=\boldsymbol{\Sigma}$ for all $i, j, k$ and $l$ allows the $d$ repeated measures to be correlated in any pattern, since $\boldsymbol{\Sigma}$ is completely general. The number of parameters which should be estimated in this covariance structure is $d(d+1) / 2$ and it is large in comparison with compound symmetry, where only two parameters should be estimated. A more general multivariate approach is the linear mixed model approach (LMM), which allows us to assume different covariance structures with less parameters to be estimated. An LMM model is also appropriate when there is heterogeneity of $\Sigma$ 's between the treatments.

\section{Results}

The estimation of model and variance-covariance parameters was calculated by the SPSS Repeated procedure and Linear mixed models procedure. For the colour variables we compared the results of two different repeated error covariance structures: compound symmetry and unstructured. In other words, we compared the results of the univariate approach with the results of the multivariate approach (Table 4). As the Maulchy's test showed, we have to use unstructured variancecovariance for the colour variables and compound symmetry for fruit firmness. But the results of the hypothesis testing are quite similar in both approaches for all variables.

Table 3 shows the results of F-tests. The factor time plays the most important role in explaining the differences between the means of all three colour variables of cherries. There is no interaction between the factors cultivar and GA3 
treatment, all the other interactions and main effects are significant. In the further analysis, we tested the different contrasts to explain the interaction and establish the significant differences between different levels of factors (Table 4). Table 4 gives only the significance of the differences of means, their values can be obtained from Figure 4, which present marginal means for the control and for the GA3 treatment showing the time-related changes of brightness, green/red and blue/yellow for each cultivar separately. The mean values of brightness are higher for $G A 3$ treated cherries, but the differences between means are significant only for the dates 3 and 4 for the cv. 'Van' and for the dates 4 and 5 for the cv. 'Sunburst'. In the case of the variable Green/Red the GA3 treatment generally gives lower values, but the differences are significant only for dates 3 and 4 for 'Van' and for date 4 for 'Sunburst'.

Most differences are significant for the variable Blue/Yellow: almost all the period for 'Van' and the last three dates for 'Sunburst'.

These results show that, to some extent, the GA3 treatment causes a little slower ripening of cherries in both cultivars and that the ripening process of 'Van' differs slightly from the ripening process of 'Sunburst'.

The results of F-tests for fruit firmness show that we can reject the hypothesis for main effects of the within-unit factor side and for main effects of the betweenunit factor cultivar. The means of firmness are otherwise systematically higher for the cherries treated with GA3 than for those, which are not, but the variability of data is too high to allow them to be significant.

Tabele 3: The results of $F$ test given by the linear mixed model analysis considering unstructured error variance-covariance for the three colour variables and compound symmetry for the firmness.

\begin{tabular}{|c|c|c|c|c|c|c|c|c|}
\hline \multirow[b]{2}{*}{ Source } & \multicolumn{2}{|c|}{ Brightness } & \multicolumn{2}{|c|}{ Green/Red } & \multicolumn{2}{|c|}{ Blue/Yellow } & \multicolumn{2}{|c|}{ Firmness } \\
\hline & $\mathrm{F}$ & $\mathrm{p}$ & $\mathrm{F}$ & $\mathrm{p}$ & $\mathrm{F}$ & $p$ & $\mathrm{~F}$ & $\mathrm{p}$ \\
\hline GA3 & 6,5 & $0,011^{*}$ & 4,0 & $0,045^{*}$ & 11,9 & $0,001 *$ & 3,8 & 0,087 \\
\hline Cultivar & 18,1 & $0,000^{*}$ & 18,0 & $0,000 *$ & 18,9 & $0,000 *$ & 6,2 & $0,037 *$ \\
\hline Date & 1541,6 & $0,000^{*}$ & 2814,9 & $0,000 *$ & 1100,4 & $0,000^{*}$ & & \\
\hline (Side) & & & & & & & 21,2 & $0,000^{*}$ \\
\hline Cultivar*GA3 & 0,4 & 0,550 & 0,2 & 0,665 & 1,4 & 0,231 & 0,07 & 0,797 \\
\hline $\begin{array}{l}\text { Cultivar*Date } \\
\text { (Cultivar*Side) }\end{array}$ & 53,5 & $0,000^{*}$ & 15,9 & $0,000 *$ & 15,8 & $0,000^{*}$ & 1.1 & 0,336 \\
\hline GA3*Date & 10,7 & $0,000 *$ & 30,6 & $0,000^{*}$ & 15,9 & $0,000^{*}$ & 0 & \\
\hline $\begin{array}{l}\text { GA3 }{ }^{*} \text { Date }^{*} \text { Cultivar } \\
\left.\text { (GA3*Side }{ }^{*} \text { Cultivar }\right)\end{array}$ & 6,8 & $0,000^{*}$ & 6,3 & $0,000 *$ & 6,5 & $0,000^{*}$ & 2,7 & 0,068 \\
\hline
\end{tabular}



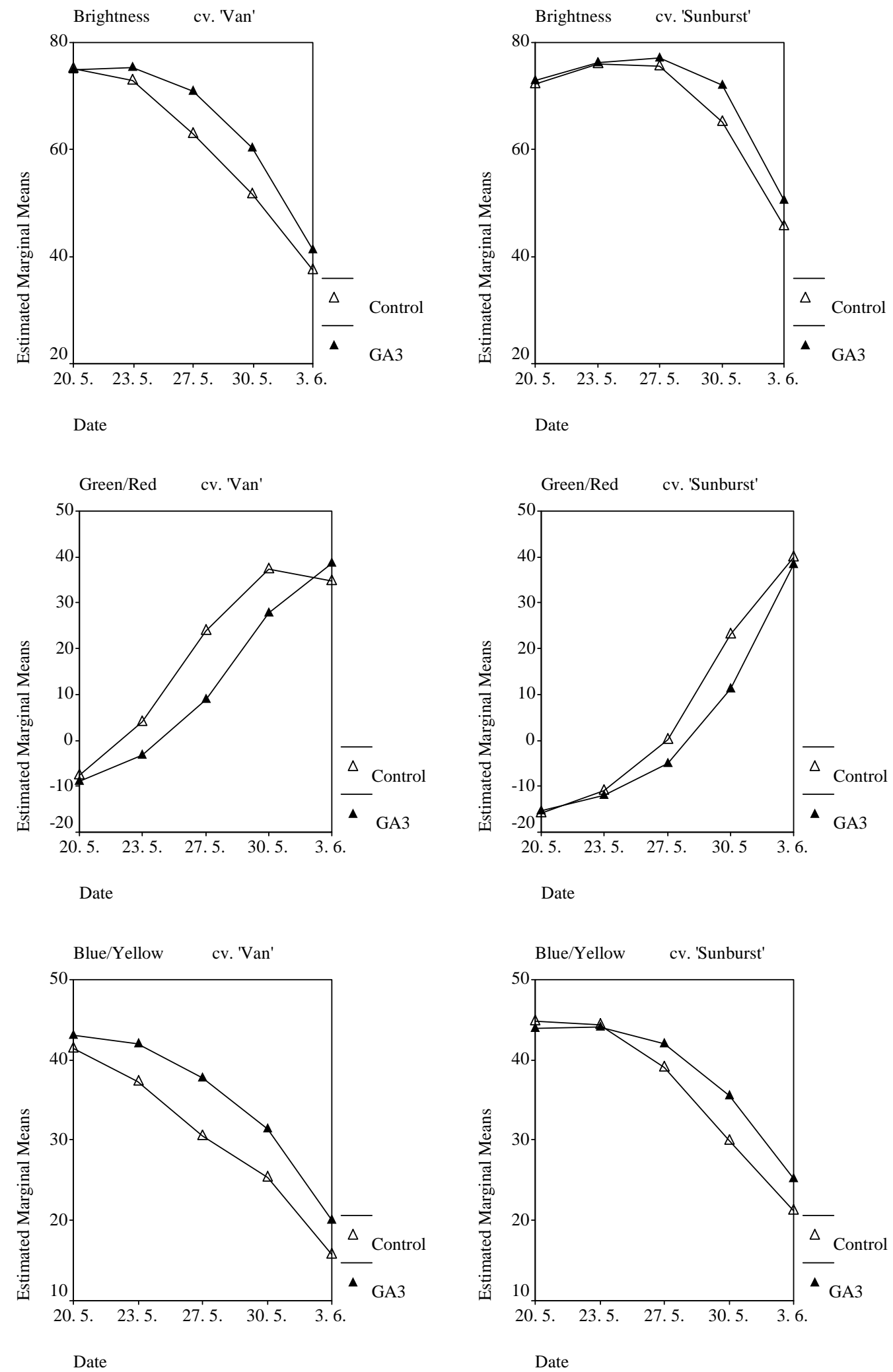

Figure 4: The marginal means for the control and for the GA3 treatment showing the time-related changes in brightness, green/red and blue/yellow for each cultivar separately. 

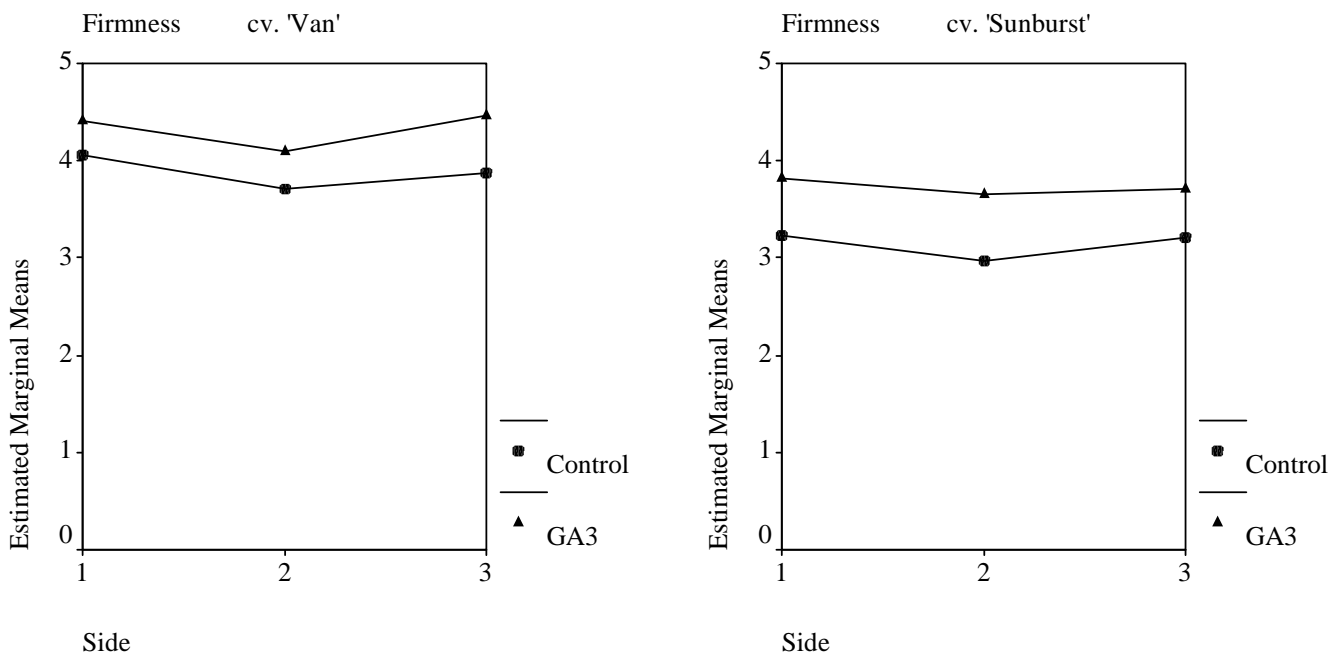

Figure 5: The marginal means for the control and for the GA3 treatment showing the time-related changes in firmness for each cultivar separately.

Table 4: The significance of differences between the means of colour and firmness variables between GA3 and Control, for each cultivar and for each date separately.

\begin{tabular}{|c|c|c|c|c|c|c|c|c|c|c|c|c|}
\hline & \multicolumn{4}{|c|}{ Brightness } & \multicolumn{4}{|c|}{ Green/Red } & \multicolumn{4}{|c|}{ Blue/Yellow } \\
\hline & \multicolumn{2}{|l|}{ 'Van' } & \multicolumn{2}{|c|}{ 'Sunburst' } & \multicolumn{2}{|l|}{ 'Van' } & \multicolumn{2}{|c|}{ 'Sunburst' } & \multicolumn{2}{|l|}{ 'Van' } & \multicolumn{2}{|c|}{ 'Sunburst' } \\
\hline & UN & CS & UN & CS & UN & CS & UN & CS & UN & $\mathrm{CS}$ & UN & $\mathrm{CS}$ \\
\hline Date 1 & 0,930 & & 0,622 & & 0,720 & & 0,883 & & 0,261 & & 0,557 & \\
\hline Date 2 & 0,348 & & 0,941 & & 0,092 & & 0,750 & & $0,005^{*}$ & $*$ & 0,855 & \\
\hline Date 3 & $0,006^{*}$ & $*$ & 0,317 & & $0,002 *$ & $*$ & 0,102 & & $0,000 *$ & $*$ & $0,046^{*}$ & \\
\hline Date 4 & $0,002 *$ & $*$ & $0,000^{*}$ & $*$ & $0,023^{*}$ & $*$ & $0,001 *$ & $*$ & $0,000 *$ & $*$ & $0,001 *$ & $*$ \\
\hline Date 5 & 0,123 & & $0,010^{*}$ & $*$ & 0,308 & & 0,580 & & $0,013 *$ & & $0,008^{*}$ & $*$ \\
\hline
\end{tabular}

UN unstructured variance-covariance; CS compound symmetry;

\section{Conclusions}

Two different approaches for the factorial-repeated-measures experiment were used and discussed for the data analysis of four variables describing cherry ripeness and firmness. From theoretical point of view, the multivariate approach is appropriate for the three colour variables and the univariate approach for the fruit firmness. The results show to some extent that the GA3 treatment causes slightly slower ripening of cherries in both cultivars and that the ripening processes of 'Van' and 'Sunburst' differ slightly. The means of fruit firmness are otherwise systematically higher for the cherries treated with GA3 than for non-treated ones, but the variability of data is too high to allow them to be significant. The estimates of error variance components from this experiment can be important information for further similar experiments planning. 


\section{References}

[1] Choi, C., Wiersma, P.A., Toivonen, P., and Kappel, F. (2002): Fruit growth, firmness and cell wall hydrolitic enzyme activity during development of sweet cherry fruit treated with gibberellic acid (GA3). Journal of Horticultural Science \& Biotechnology, 77, 615-621.

[2] Demirsoy, L. and Bilgener, S. (1998): The effect of preharvest chemical applications on cracking and fruit quality in 0900 'Ziraat', Lambert' and 'Van' sweet cherry varieties. Acta Horticulturae, 468, 663-670.

[3] Diggle, P.J. (1988): An approach to the analysis of repeated measurements. Biomatrics, 44, 959-971

[4] Facteau, T.J., Rowe, K.E., and Chesnut, N.E. (1985): Response patterns of gibberellic acid-treated sweet cherry fruit at different soluble solids levels and leaf/fruit ratios. Scientia Horticulturae, 27, 257-262.

[5] Kappel, F. and MacDonald, R.A. (2002): Gibberellic acid increases fruit firmness, fruit size, and delays maturity of 'Sweetheart' Sweet Cherry. Journal American Pomological Society, 56, 219-222.

[6] Lindsey, J.K. (1999): Models for repeated measurements, Sc. Ed.. New York: Oxford University Press Inc.

[7] Littell, R.C. (1989): Statistical analysis of experiments with repeated measurements. HortScience, 24, 37-40.

[8] Rencher, A.C. (1995): Methods of multivariate analysis. New York: John Wiley \& Sons, Inc.

[9] Wiener, B.J. (1970): Statistical Principles in Experimental Designs. International Student Edition, McGraw-Hill, Mladinska knjiga Ljubljana. 